\title{
Haar-features training parameters analysis in boosting based machine learning for improved face detection
}

\author{
Vikram Mutneja $^{1^{*}}$ and Satvir Singh ${ }^{2}$ \\ Associate Professor, Department of Electronics \& Communication Engineering, Shaheed Bhagat Singh State \\ University, Ferozepur, Punjab-152004, India ${ }^{1}$ \\ Associate Professor, Department of Electronics \& Communication Engineering, I.K. Gujral Punjab Technical \\ University, Kapurthala, Punjab-144603, India ${ }^{2}$ \\ Received: 15-April-2021; Revised: 13-July-2021; Accepted: 19-July-2021 \\ (C)2021 Vikram Mutneja and Satvir Singh. This is an open access article distributed under the Creative Commons Attribution (CC \\ BY) License, which permits unrestricted use, distribution, and reproduction in any medium, provided the original work is properly \\ cited.
}

\begin{abstract}
Haar features have been used in most of the works in literature as key components in the task of object as well as face detection. Training of Haar features is an important step in the development of overall machine learning based face detection system. In this work, we have done investigation in the variations of a number of training parameters during AdaBoost based machine learning of Haar features with respect to the size of training images. A number of observations have been drawn based on the variations of these parameters during the training process. Training parameters, true detection rate and figure of merit have been used as weighing parameter. These parameters have been used in the formation of detection cascade for the improvement in the original AdaBoost framework used for machine learning of Haar features. Statistical analysis based on variations of training parameters has been done for selection of efficient learners from a large pool of available features which further are cascaded to make the strong classifiers. We have been able to achieve the maximum percentage reduction in training time of 47.20 corresponding to training image's size of $25 \times 25$ pixels with the help of statistical screening of Haar features prior to AdaBoost. The proposed system has been developed using training dataset from Center for Biological \& Computational Learning $(C B C L)$ and successfully tested on facial images from datasets. The datasets considered were WIDER FACE Detection Benchmark, Annotated Faces in the Wild (AFW) and Face Detection Dataset and Benchmark (FDDB). The results achieved were promising in terms of training time computed in the range of 0.31-2.83hrs. The maximum detection rate is $96.21 \%$ and minimum detection time is $16.95 \mathrm{~ms}$.
\end{abstract}

\section{Keywords}

Haar features training, AdaBoost, Machine learning, Statistical analysis, Face detection, Features reduction.

\section{Introduction}

There is very strong motivating factor for doing research in the field face biometrics for the purpose of authentic access to various resources. In the prevalent situation of pandemic COVID-19, remote logging as well as contactless authentication systems have emerged out to be of great importance. There has been a tremendous rise in usage of video cameras and surveillance systems, thereby giving more impetus to research in the domain of face detection and facial image processing systems which is mostly machine learning based.

Features are an important constituent used in the framework of classification task.

*Author for correspondence

919
The Haar classifiers have been used as prominent features since last two decades in the most of the works in the field of object detection, particularly in the domain of face detection. Figure 1 shows the shape and types of different types of rectangular Haar classifiers and their application in localizing various facial parts such as nose, lips and eyes. The pioneer work in this domain was done by seminal Viola-Jones face detection algorithm $[1,2]$.

In the features training process for the generation of eligible candidates from the large pool of classifiers for the task of detection. It is always desirable to achieve the required performance of the classification task with the reduced set of features. A significant amount of research $[1,3,4,5]$ has been done in the field of statistical analysis of features and modifications of the selection criteria of features in 
AdaBoost algorithm for achieving better performance of object detection.

Haar features training parameters can be used either in process of feature selection based on statistical analysis or be used as weights of decision stumps in AdaBoost. The statistical properties can give insight about the quality of Haar features so that it can be straightway selected or rejected. During the process of applying Haar wavelets on an input image, all the pixels are scanned by the growing size of rectangular Haar classifiers so as to detect the faces of different dimensions present in the image. This process of scaling of Haar classifiers eventually results in a large population of Haar classifiers generations. Figure 1 shows the application of some of the selected Haar features of the facial images of size $24 \times 24$ pixels.

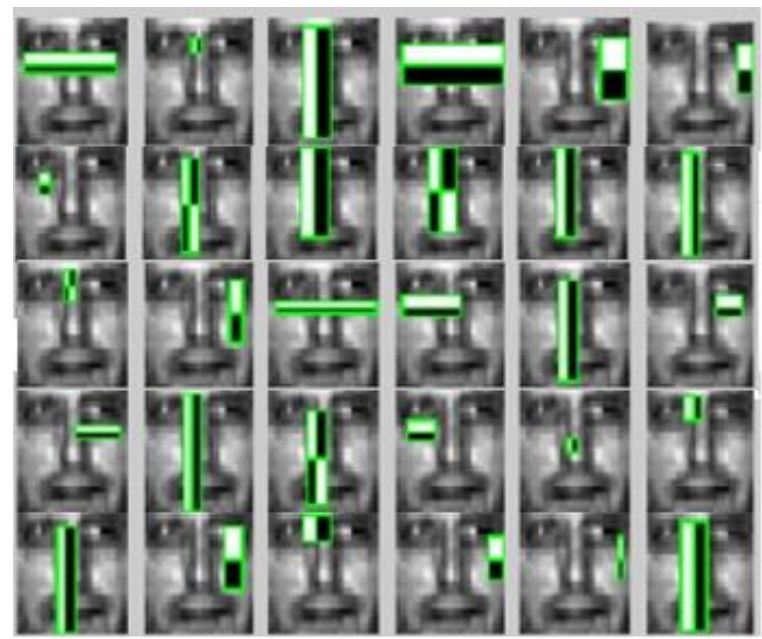

Figure 1 Application of Haar features in a training facial image

This work focuses on analysis of training parameters which have been introduced in the machine learning process of Haar features. The methodology is based on framework of Graphical Processor Unit (GPU) accelerated boosting based features training and face detection done in our previous work [6, 7]. Detailed training analysis has been done for variations of these training parameters on inter-class as well intra-class basis with respect to size of training images.

\section{Literature survey}

A lot of work has been done in the field of Haar features machine learning techniques. Haar features were first introduced by Papageorgiou et al. [8] in 1998. A number of works afterwards used the Haar features and proposed their extension in task of object detection such as by Lienhart and Maydt [9], Viola and
Jones [1] including the seminal work done in the area of face detection by Viola and Jones [2].

Saha and Démoulin [10] introduced compact and efficient version of Haar features as Aggregated LOcal Haar (ALOHA). It is used for grouping the pixels within a patch centered on a particular key point. A lot of work has been done in the statistical analysis of Haar-like features for efficient training.

Nirmalkar and Janghel [11] worked on Haar features selection using statistical techniques in conjunction with the boosting to reduce the training time with significant gains in the accuracy.

Zhang et al. [12] worked on the systematic design of a collection of rectangular templates. It could be applied to shape model of up-right human body for the pedestrian detection by using specialized Haar-like features modified to incorporate low level measurements. The purpose was to characterize human body parts, handling variations in clothing and environmental factors, tested on INRIA and Caltech pedestrian datasets.

Many works have targeted the analysis of training of Haar-features, modification in the AdaBoost, training framework and implementation of different types of applications.

Wang [13] worked on analysis and developing the complete system based on Viola-Jones algorithm, with the additional step to handle detection redundancy using a robust argument.

Murphy et al. [14] constructed framework constituting a neural network and truncated Viola-Jones cascade so as to improve false negatives.

Qezavati et al. [15] worked on partially covered face detection in low resolution surveillance videos of traditional Middle Eastern clothing such as headscarf, using a Haar cascade and Local Binary Patterns Histogram (LBPH) for feature extraction and the Support Vector Machine (SVM) for face classification.

Many researchers have reported the features set reduction by using statistical analysis. Chung et al. [16] reported to achieve features set reduction to $20 \%$ without affecting the detection accuracy by worked on removal of redundant Haar-features identified based upon similarity with previously selected features. 
Mutneja and Singh [17] worked on the analysis of Haar features training parameters with respect to the varying size of training images. A number of parameters in Haar features training have been proposed and investigation of their effect on training process has been done and finally a configuration for improvised face detection has been proposed.

Jin et al. [18] proposed the feature binding strategy for making features group bearing different weight coefficients determine the object and its location. Authors contend enhancement of the detection accuracy and speed object detection.

Rusia et al. [7] suggested an attendance monitoring system based on face detection and recognition using Haar-like features and Local Binary Patterns (LBP) histogram with face identification accuracy of 81.6 percent.

Lin et al. [19] proposed Haar-like features-based iris localization from the low-quality images using the AdaBoost algorithm, reported acceptable accuracy and processing speed for their cascade classifier.

In [20] authors developed face detectors using Haarlike and local binary pattern features, based on their number of uses at each stage of training. The study of training parameters like False Alarm Rate (FAR) and True Positive Rate (TPR) has been done in MATLAB's trainCascadeObjectDetector function.

Pant and Sthapit [21] presented machine learning based method using Haar Cascade for face detection, deep Convolution Neural Network (CNN) layers for feature extraction and Recurrent Neural Network (RNN) for recognition of micro-facial expression analysis.

Haar cascade has been used not only in face detection, but also very specialized fields of object detection such as detection and recognition of electronics components.

Mordvintseva and Belitsky [22] presented a solution for rapid classifying and sorting electronic components. So that some of electronic components which contain mercury, cadmium and chromium were identified and preserved to be recycled properly.

Odaudu et al. [23] worked on satellite image processing by introducing Differential Evolution (DE) algorithm for selection and mapping of features to Haar cascade machine learning classifier.
Wang et al. [24] proposed compressive Haar transforms based graph pooling through a cascade of operations following a sequence of clustering's of the input graph. The objective was using a HaarPooling layer to transform an input graph to output graph with sparse representation-based feature vector of uniform size without losing its structural information.

In [25-27], authors proposed face detection by scaling upHaar features while parsing the image and computing the pixel sum each time using an integral image. Afterwards detected facial images were compared with stored training samples by using the Euclidean distance for face recognition.

As per literature survey, it has been found that most of the work since past two decades in the domain of machine learning based face detection has been based on Haar features. Their training has been performed using AdaBoost or its variants. Haar features, because of their versatility have been used not only in face detection, but also in other types of object detections $[19,22,28]$ as discussed above. Therefore there is credible amount of significance of carrying the research in domain of Haar features, their training and using it in machine learning based face detection.

\section{Experimental setup}

Proposed work has been implemented in MATLAB version 2013b environment using libraries of its parallel computing toolbox on WINDOWS 10 Pro 64bit host operating system. It is with the GeneralPurpose Graphical Processing Unit (GPGPU) programming for parallelization done of the NVIDIA GeForce GT740M GPU.

\section{Proposed method for machine learning of Haar features}

Machine learning of Haar features is an essential step in the proposed system, which has been done by using example images of different size. The training database considered from Center for Biological \& Computational Learning (CBCL) at Massachusetts Institute of Technology (MIT) [29]. It contains 2429 faces and 4547 non-faces images of base size of $19 \times 19$ pixels. The training databases of various sizes (in the range from $14 \times 14$ to $30 \times 30$ pixels) have been generated from base database by scaling. The proposed AdaBoost based method comprises of statistical analysis and modified training process. Many parameters were associated with the training which has been discussed in the following subsections. 


\subsection{Training parameters}

In depth analysis of the machine learning process of Haar features through AdaBoost has been done. Number of training parameters have been introduced and investigated as discussed below. These parameters are calculated and stored for each of the selected weak classifiers.

\subsubsection{Positional and shape related parameters}

These are the six main parameters named as $x, y, w, l$, cType and $A$. These are characterized as a Haarclassifier position and shape. $x$ and $y$ are cartesian coordinates specifying the position of Haar-classifier, $w$ and $l$ specify width and length respectively of a selected weak classifier type cType (1-5), and $A$ its area which is calculated as product of width $(w)$ and length $(l)$.

4.1.2Threshold related parameters

i) tpMin, tpMax, tpMean specifies minimum, maximum and mean values respectively of thresholds computed for positive training images.

ii) $L_{L}, \quad U_{L}$ specifies lower and upper limits respectively, of threshold computed for active window of a specific weak classifier.

iii) ThRange represents a threshold range, which is equal to size of screening window of threshold of a weak classifier which is used to classify an image window under consideration as facial or non-facial image window, computed (Equation 1).

ThRange $=\left(U_{L}-L_{L}\right)<B W$

iv) $B W$ is a bandwidth of selected weak classifiers, measured (Equation 2)

$B W=t p M a x-t p M i n$

4.1.3Detection related parameters

i) $T_{P}$ is the number of true face detections, while $T_{N}$ specifies non-face detections.

ii) $F_{P}$ is the number of false face detections and $F_{N}$ number of false non-face detections.

iii)TTD , TFD are Total Number of True Detections and Total Number of False Detections respectively, including the facial as well as non-facial images (Equation 3 and 4).

$T T D=T_{P}+T_{N}$
$T F D=F_{P}+F_{N}$

iv)FOM represents Figure of Merit, computed through the Equation 5.

$F O M=\frac{T_{P}+T_{N}}{F_{P}+F_{N}}$

4.1.4Detection rate related parameters

i) $T P R$, measured through the Equation 6 .
$T P R=\frac{T_{P}}{\text { TotalNumImages }}$

ii) True Detection Rate (TDR) calculated through the Equation 7.

$T D R=\frac{T_{P}+T_{N}}{\text { TotalNumImages }}$

iii) FDR represents False Detection Rate is given as per the following Equation 8.

$F D R=\frac{F_{P}+F_{N}}{\text { TotalNumImages }}$

Where the TotalNumImages is total number of training images including faces as well non-faces.

\subsection{Statistical analysis of training parameters}

We have used the statistical analysis of features as prescreening stage before the AdaBoost to enhance the time efficiency of haar features training. The statistical criteria have been computed based upon threshold values belonging to feature vectors of both classes i.e. faces and non-faces. The following criteria have been used as binary decision based on interclass separation or overlap.

4.2.1Interclass distance

Feature threshold values were computed as described in the steps of the training process. A good feature should be able to create the maximum inter-class margin and minimum intra-class scattering. Taking this into account following criteria has been set for the direct selection or rejection of features.

$C 1(\max )<=C 2(\min )$

$C 2(\max )<=C 1(\min )$

$C 2(\min )>=C 1(\min ) \operatorname{and} C 2(\max )<=C 1(\max )(11)$

$C 1(\min )>=C 2(\min )$ and $C 1(\max )<=C 2(\max )$

Where

C1 (max): Maximum value of threshold computed in the feature vector of first class (Faces)

C2 (max): Maximum value of threshold computed in the feature vector of second class (non-Faces)

Cl ( $\mathrm{min}$ ): Minimum value of threshold computed in the feature vector of first class (Faces)

C2 ( $\min$ ): Minimum value of threshold computed in

If Equations 9 or 10 have been satisfied, the feature is directly considered as good feature i.e., it need not to be passed to the AdaBoost. Features satisfying Equations 11 or 12 are straightway declared as unfit candidates as they belong to the category in which feature vectors overlap. 
However, the criteria as per Equations 9, 10, 11 and 12 has a constraint that the accuracy of classifiers may be dependent upon the quantity and types of images. It consists of training database containing of positive and negative examples. The large variability and size of database would be required to generate the true representations of constituent classes in terms of its calculated feature vectors. The results obtained though the Equations 9, 10, 11 and 12 may not be satisfactory in case feature vector values turn out to be superficial owing to inadequacy being reflected in the training dataset in terms of lack of quantity or variability of example images.

4.2.2Weak learner selection criteria

The values of threshold limits of training errors i.e., Total Error $\left(E_{t}\right)$ and Positive Error $\left(E_{p}\right)$ have been set as 0.6 and 0.06 respectively. These are 0.5 and 0.05 in original AdaBoost, and additional limit on parameter $T D R$ has been set during the training process as shown in Equation 13.

$\left(E_{t}<=0.6\right) \operatorname{and}\left(E_{p}<=0.06\right)$ and $(T D R<=0.5)$

\subsection{Training process steps}

The proposed training process of Haar features based on statistical analysis and AdaBoost has been done as per the following steps:

a) Generation of pool of rectangular Haar features of various types and size. As the Haar features have grown in size from minimum size 2 by 2 pixels up to a maximum based on the size of training images for the large pool of Haar feature generation.

b) Application of Haar features one by one on training images comprising of faces as well as non-faces.

c) Calculation of the feature's value which is done as the difference between the sums of the intensity values of pixels lying in white rectangle and the black rectangle region.

d) Calculation of various training parameters as discussed.

e) All the Haar features were processed through AdaBoost based machine learning phase. It is in the selection of classifiers which are termed as weak learners and have to be subsequently cascaded to form strong classifiers through the second phase of AdaBoost.

f) Statistical analysis as discussed in previous subsection was used as a screening stage to filter out ineligible classifiers from the set of weak learners.

g) Analysis of various training parameters has been carried out (refer section 5.1). Experimentation has been done using different parameters such as TDR and FOM. It is considered as the weights of classifiers in the second phase of AdaBoost for cascading of weak learners to generate the strong classifiers. So as to form the detection cascade.

h) The process has been repeatedly carried for training datasets comprising of images of size in the range of $14 \times 14$ to $30 \times 30$ pixels.

\subsection{Face detection algorithmic steps}

Following algorithmic steps have been implemented for the development of the proposed face detection method:

a) Generation of sub-images from the input image.

b) Application of detection cascade on the picked image window.

c) Calculation of result of application of the weak classifiers on the particular sub-image, i.e., to screen the picked image window through the array of weak classifiers. It is, so to generate the binary array of classification results as, face or non-face.

d) The product of generating binary array and weighing a parameter array has been computed. Various training parameters such as TDR, FOM have been experimented to use as weighing parameter. Weighing parameter array is generated and stored during the training time only to be used during the detection process.

e) Array of real numbers computed in the previous step is grouped as per the dimensions of the detection cascade as per AdaBoost algorithm. It results in the final classification result at face or non-face of an image window under process.

\section{Results \& discussion}

\subsection{Observations from analysis of training} parameters

The training analysis of Haar features has been done with respect to the different parameters associated with the training. The parameters have been calculated for the features in each case. Corresponding minimum, maximum and mean values have been computed to investigate their inter-class variations with respect to the size of the training images in each case.

Figures 2, 3, 4 and 5 show the variations of true negatives, true positives, false negatives and false positives respectively with respect to the training image size. 
Vikram Mutneja and Satvir Singh

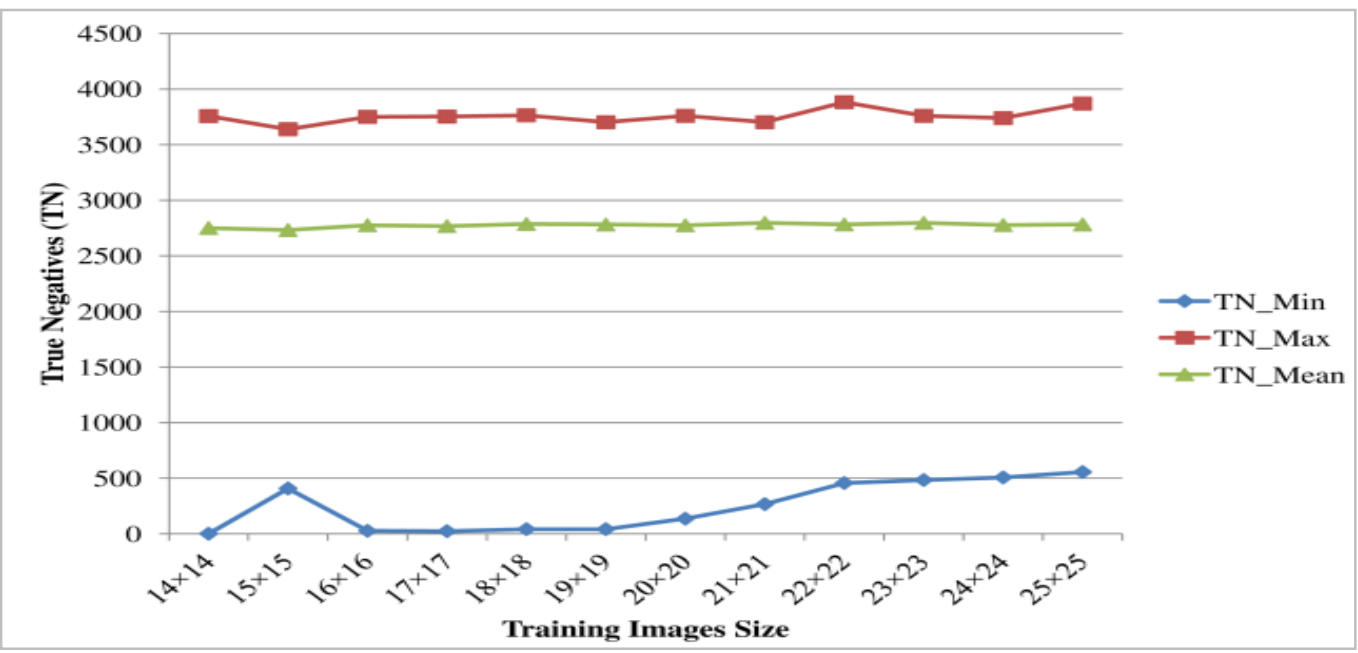

Figure 2 Comparative analysis of true negatives

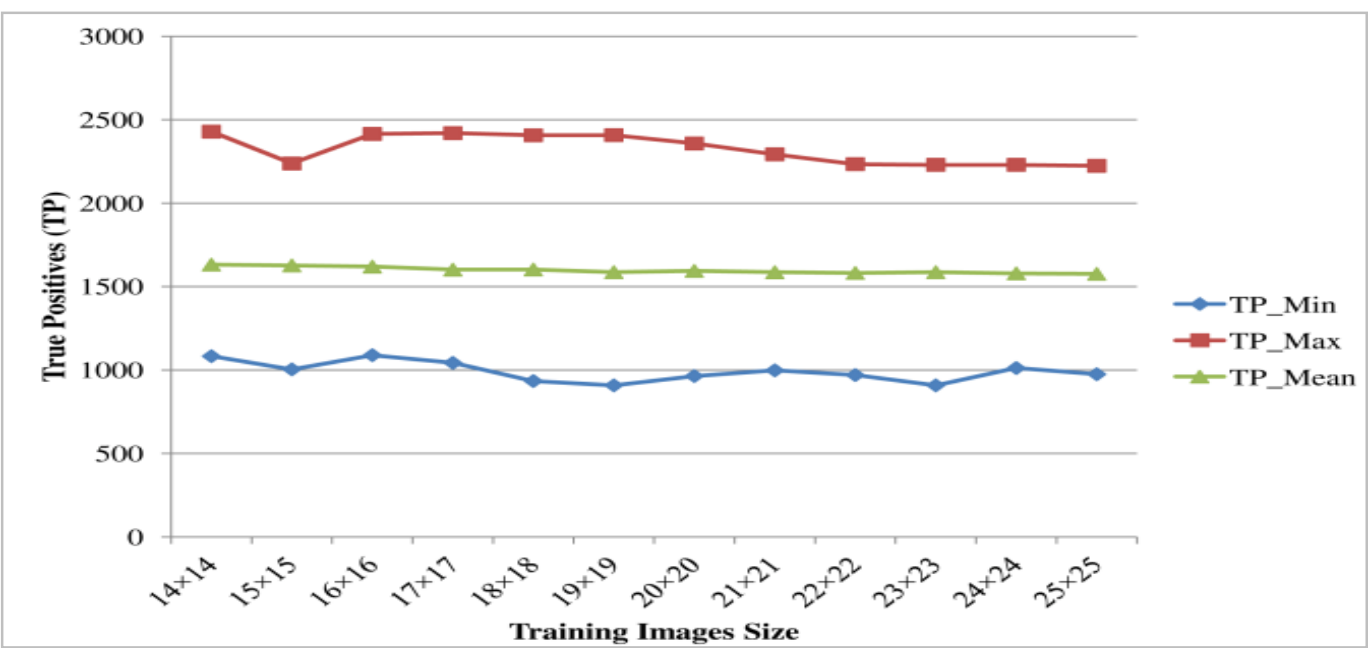

Figure 3 Comparative analysis of true positives

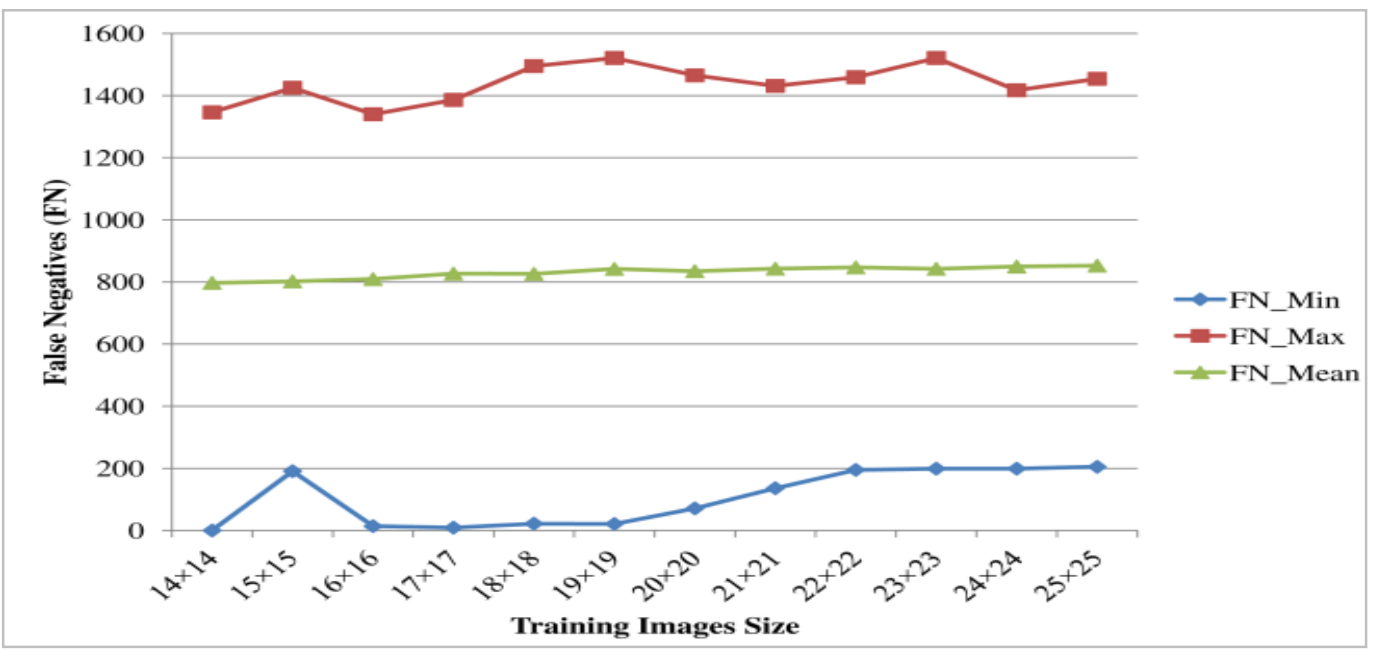

Figure 4 Comparative analysis of false negatives 


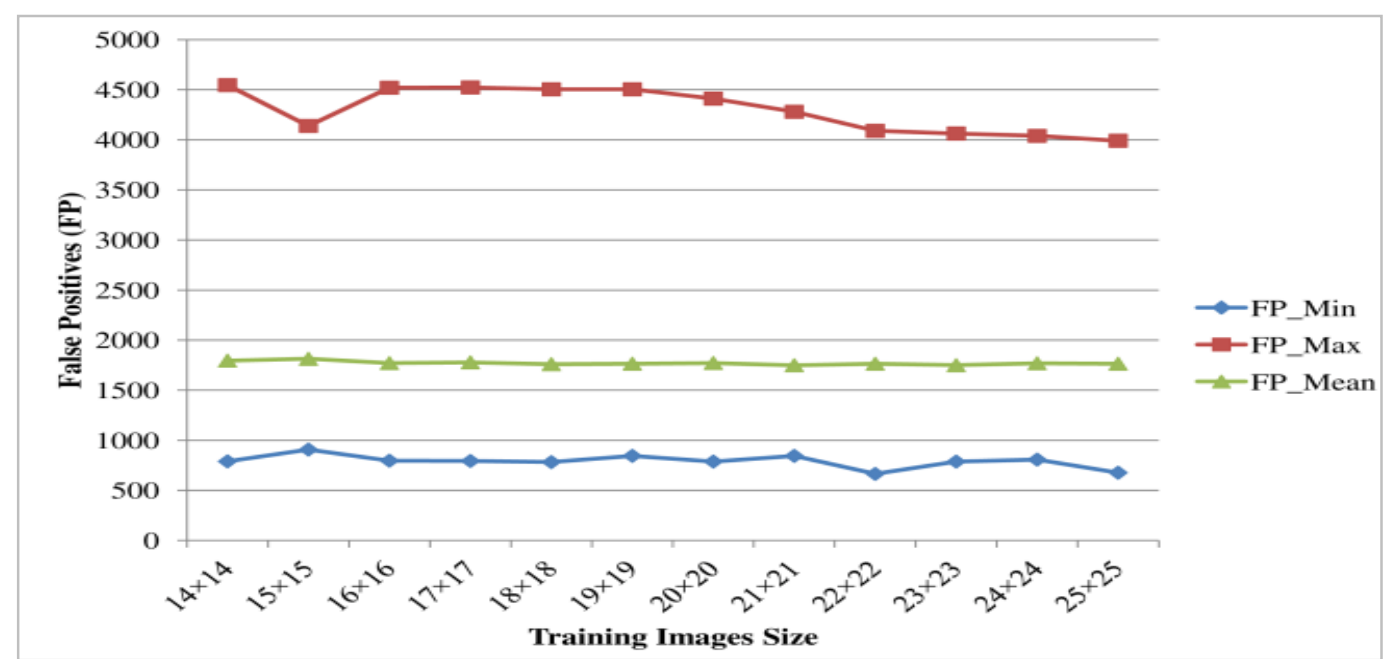

Figure 5 Comparative analysis of true positives

It is inferred from Figures 2, 3, 4 and 5, that there are only slight variations in the number of detections with the change in training image's size. Area of selected weak classifiers is an important parameter to investigate. The minimum area of selected classifiers is 2. Figure. 6 shows the variations in the parameter area with respect to the size of the training images.

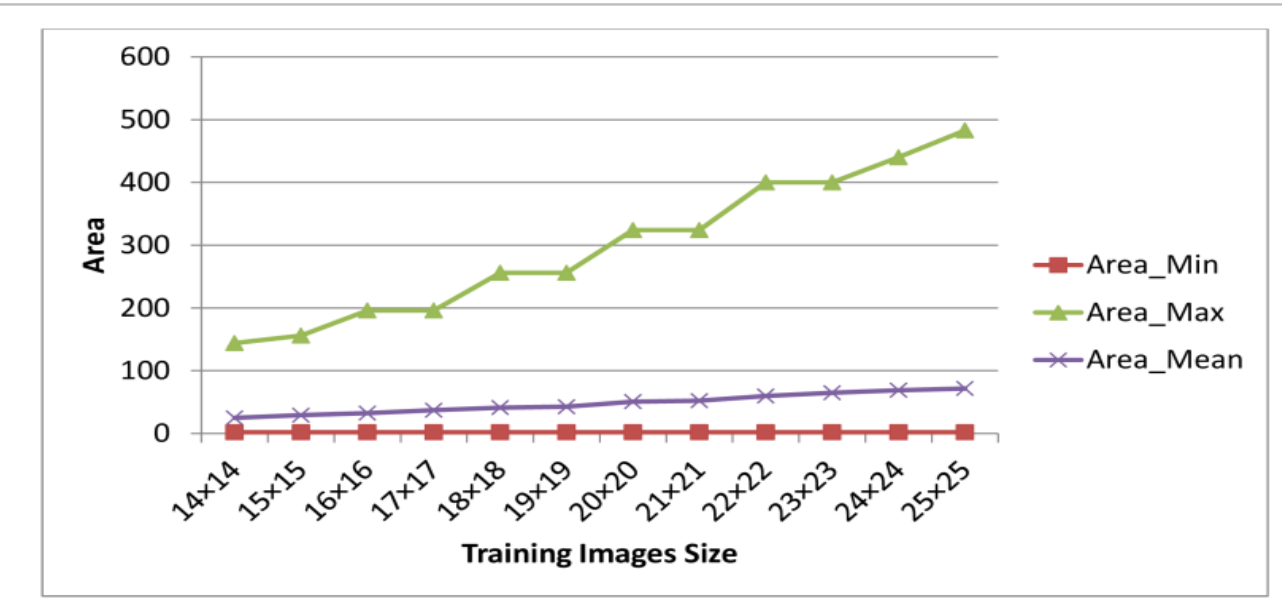

Figure 6 Variations of classifiers area (in Pixels) with respect to training image's size

It is inferred from the variations, that with the increase in the size of the training images there is a gradual, linear rise in average area while the variation in maximum area of classifiers is steeper. Following enumerates the observations made from the intra class behaviour of various training parameters:

1. The parameters can be divided into two categories based upon their impact on detection results. Firstly, true positive detections were considered and secondly, true as well as false detections. If we select the parameters, which takes into account only true positive detections such ThRange, $B W$ for detector cascade configuration, there is an increase in the true positive detection rate but at the same time, there is also increasing in the false positives. However the parameters such as FOM, which is based on true as well as false detections, results in lesser number of false positives, but at the cost of fall in the detection rate.

2. The analysis has been done with respect to the parameter Area. It has been found that good detection rate is found when used area to sort the features in descending order for the formation of detection cascade. It shows that more the area of the features, better is the ability in distinguishing the face details. 
3. Experimentation has also been done by using the combination of classifiers for configuring the detection cascade, such as ThRange, TDR, FOM, $B W$, etc. It results in a better detection rate at the cost of increase in false positives.

4. It has been observed that resultant classifiers generated by AdaBoost training process depends upon the sequence in which they are fed-in, i.e., AdaBoost doesn't always produces the same set of classifiers, but depends upon the sequence of feeding the input features. This is attributed to the fact that during the AdaBoost, the weights of example images are modified. It depends upon the accuracy level of the class being tested.

\subsection{Training time efficiency}

Training time is computed as the sum of the time taken by GPU Compute Unified Device Architecture (CUDA) kernels in the computation of thresholds in parallel and time to process the features sequentially through AdaBoost. Table 1 shows the comparison of training time for different training image's size. It shows the improvement achieved by the statistical analysis in conjunction with AdaBoost. Figure 7 presents the graphical representation of the variability of training time, from which it is inferred that the amount of training time reduction is more significant in higher size of training images, achieved maximum corresponding to training images of size $25 \times 25$ pixels.

From Table 1, it is inferred that we have been able to achieve the training time in the range of 0.31 to 2.83 hrs. It depends upon the size of training images being used. Further, we have been able to achieve the maximum percentage reduction in training time of 47.20 corresponding to training image's size $25 \times 25$ pixels with the help of statistical screening of Haar features prior to AdaBoost. However, taking care of trade-off between training time and percentage reduction in training time, the most preferable size of training images can be $18 \times 18$ pixels. As it has low training time i.e., 45.73 minutes, and also a significant percentage reduction in training time by statistical screening, which comes out to be 38.37 .

Table 2 shows the comparative analysis of training time of features. It is inferred that proposed training method has outperformed in comparison to the listed recent works of features training. It is in terms of training time efficiency.

Table 1 Training time improvement using statistical analysis and AdaBoost

\begin{tabular}{llll}
\hline & & Training time (minutes) & \\
\cline { 2 - 4 } Image size (in pixels) & AdaBoost & $\begin{array}{l}\text { AdaBoost }+ \\
\text { Statistical analysis }\end{array}$ & $\begin{array}{l}\text { Percentage } \\
\text { reduction in training time }\end{array}$ \\
\hline $14 \times 14$ & 24.49 & 18.49 & 24.47 \\
\hline $15 \times 15$ & 33.07 & 28.88 & 12.67 \\
\hline $16 \times 16$ & 41.96 & 36.61 & 12.75 \\
\hline $17 \times 17$ & 53.96 & 45.73 & 15.25 \\
\hline $18 \times 18$ & 74.20 & 45.73 & 38.37 \\
\hline $19 \times 19$ & 91.78 & 70.25 & 23.46 \\
\hline $20 \times 20$ & 107.70 & 76.69 & 28.79 \\
\hline $22 \times 21$ & 154.42 & 96.25 & 37.67 \\
\hline $23 \times 23$ & 210.83 & 115.30 & 45.31 \\
\hline $24 \times 24$ & 237.62 & 127.14 & 46.50 \\
\hline $25 \times 25$ & 274.23 & 150.53 & 45.11 \\
\hline
\end{tabular}

Table 2 Comparative analysis of training time efficiency

\begin{tabular}{ll}
\hline Method & Training time (hrs) \\
\hline BORE (2018) [30] & 8 \\
\hline TinyCNN (2020) [31] & 40 \\
\hline Fuhl (2021) [32] & 1 \\
\hline Proposed Work & $0.31-2.83$ \\
\hline
\end{tabular}




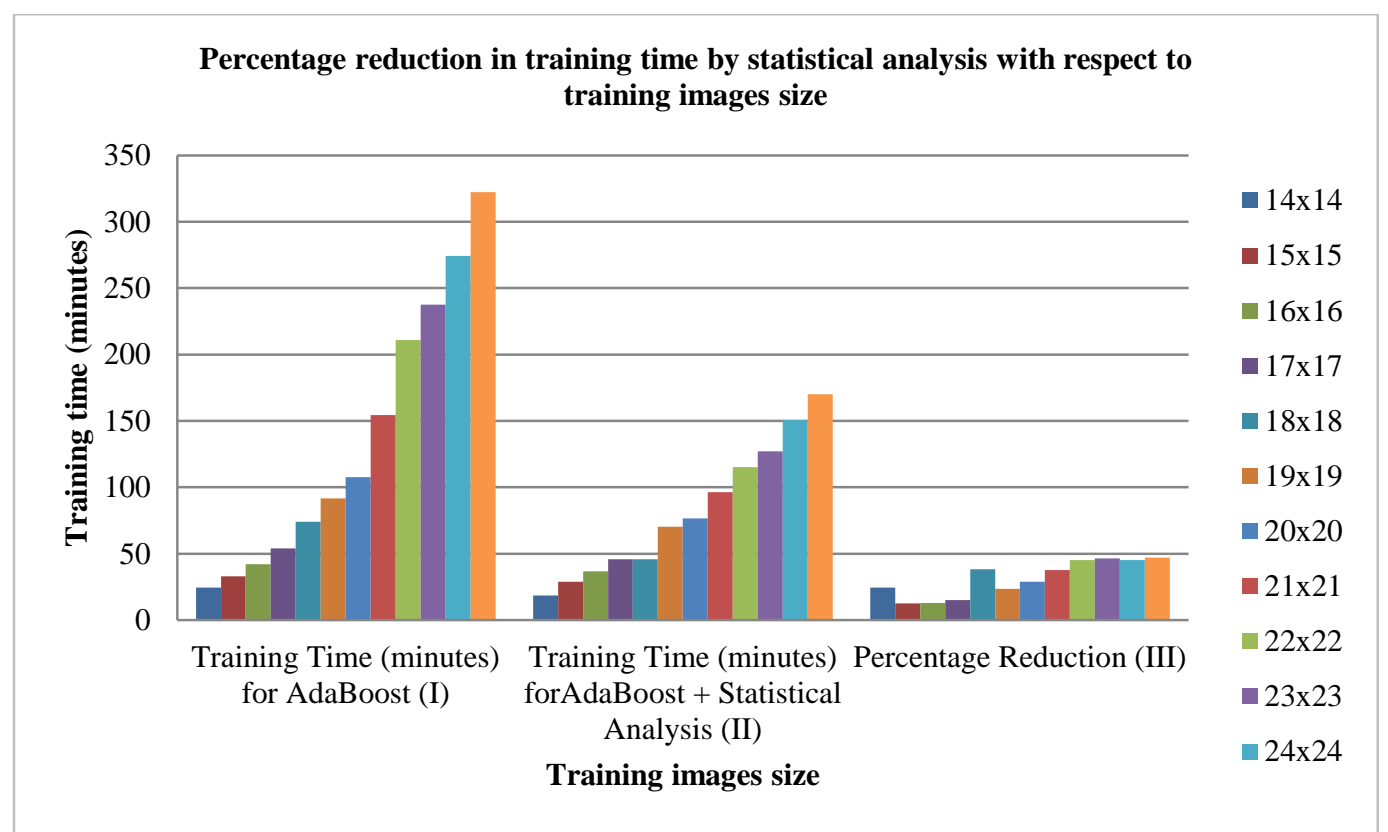

Figure 7 Percentage reduction in training time by statistical analysis with respect to training image's size

\subsection{Detection results}

We have tested the proposed system on a number of test images of varying resolution from the datasets WIDER FACE [16], AFW [17] and web faces. Figure 8 shows the application of the proposed system on some of the test images from these databases by taking TDR as weight parameter in AdaBoost algorithm.
Further, we have tested the proposed system on the Face Detection Dataset and Benchmark (FDDB) database [28] for detailed analysis of detection accuracy and time efficiency. FDDB [28] contains 2845 test images of varying resolution. Figure 9 shows the detection results on the random images from FDDB [28]. FOM has been used in this experiment, as the weighing parameter in the AdaBoost.

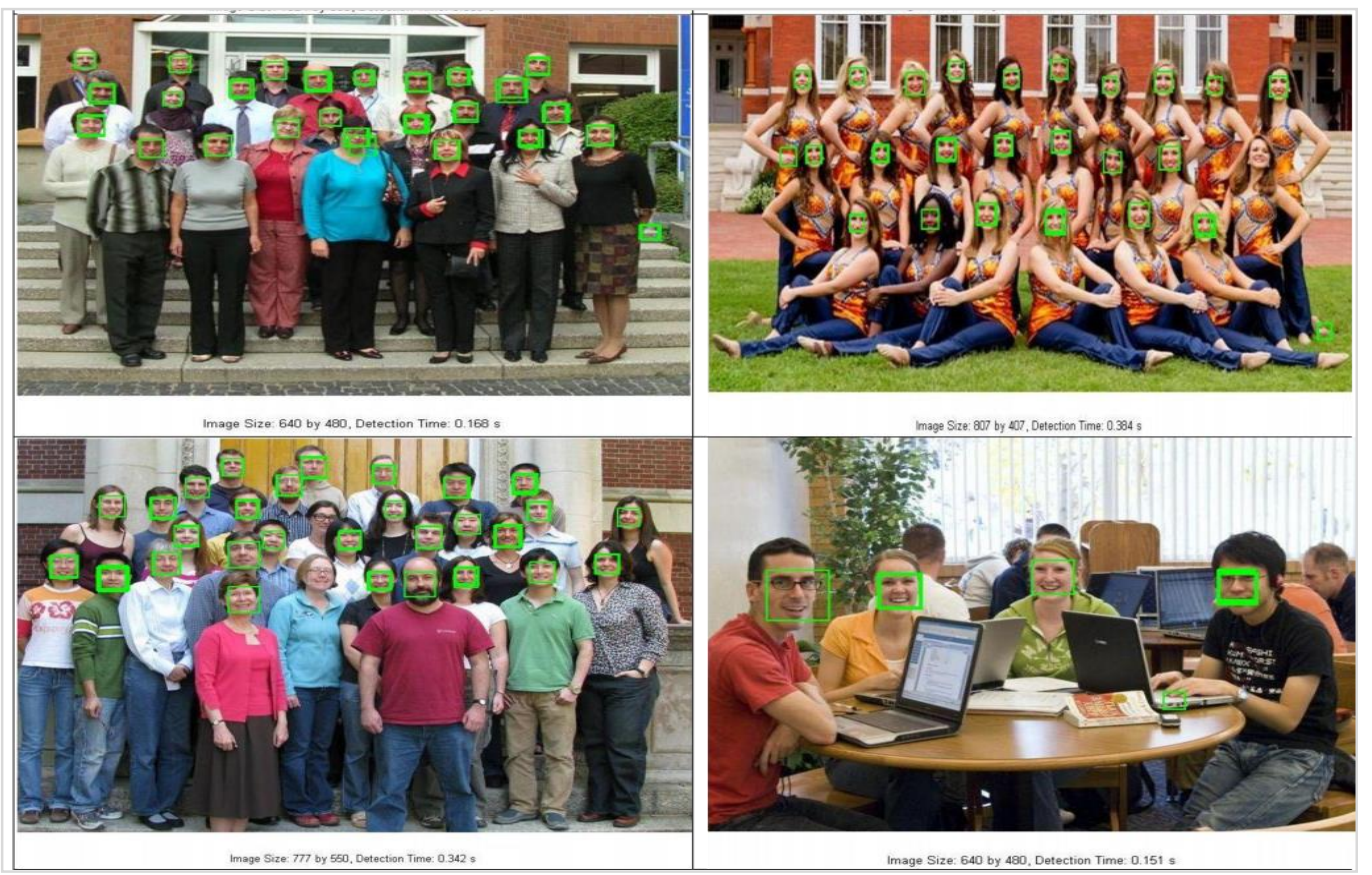

Figure 8 Example of face detection on test images from different datasets 

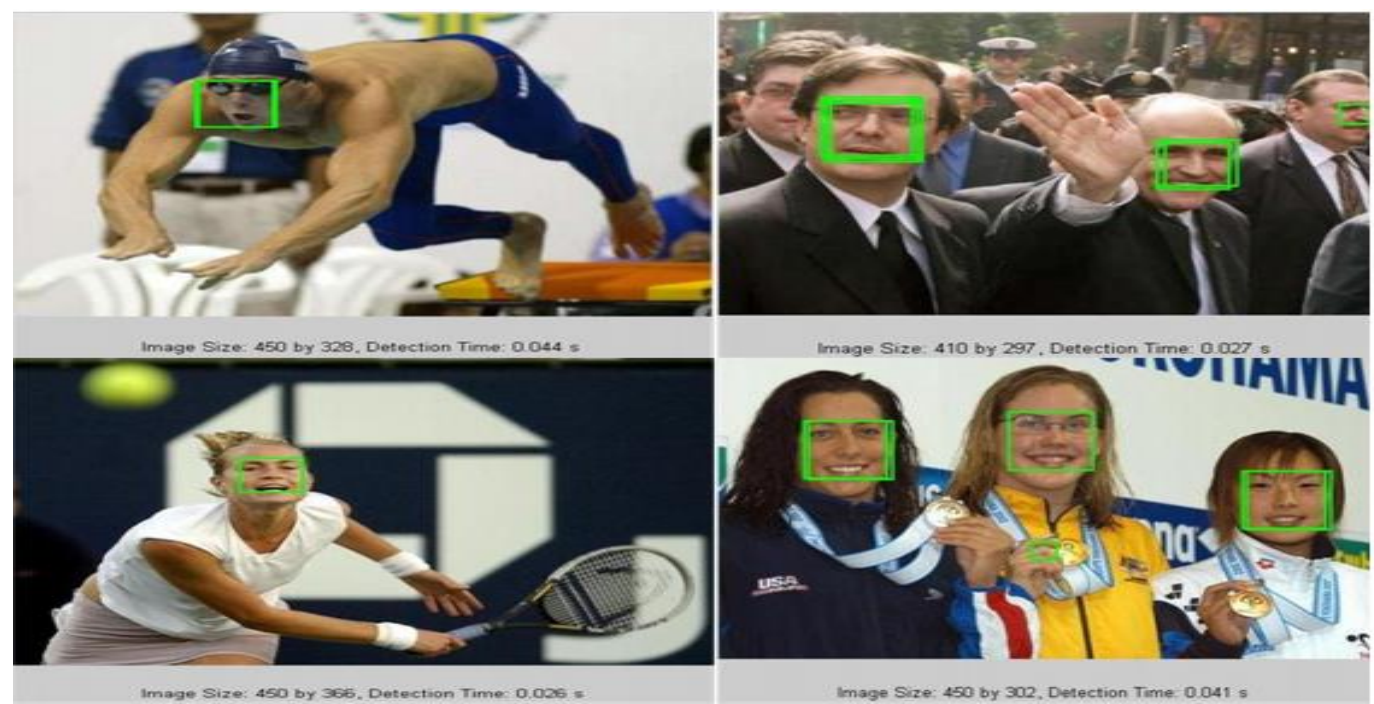

Figure 9 Results of applying proposed system on test images from FDDB dataset

The detection time in terms of processing time per pixel of the first 100 images of FDDB [28] has been investigated for the calculation of the detection time efficiency (Figure 10). We have been able to achieve minimum processing time per pixel equal to 0.004 micro-seconds, maximum of value 0.927 microseconds and mean processing time per pixel as 0.339 micro-seconds.
Table 3 shows the comparative analysis of the proposed method with some of the recent related works in the literature in terms of detection rate and detection time. Proposed method 1 has used TDR and method 2 has used FOM as weighing parameter information and evaluation of detection cascade in AdaBoost. From the comparison, it is inferred that the proposed work has outperformed with the listed works. Complete list of abbreviations is shown in Appendix I.

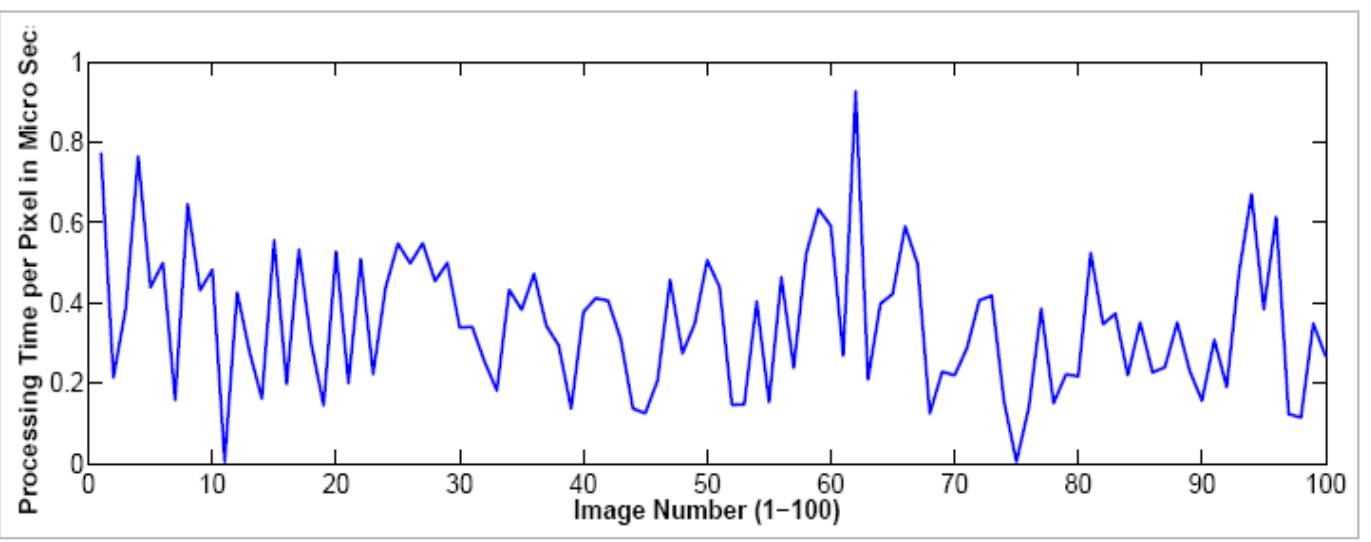

Figure 10 Plot of processing time per pixel of 100 Images from FDDB

Table 3 Comparative analysis of detection results

\begin{tabular}{lll}
\hline Method & Detection rate (\%) & Detection time (ms) \\
\hline Viola and Jones algorithm[2] & 88 & 67 \\
\hline Naido and Porle [33] & 81.91 & - \\
\hline Hou and Wang [34] & 95.41 & 770 \\
\hline Naveen and Sivakumar [35] & 90 & 17.67 \\
\hline Proposed method 1 (TDR) & 96.21 & 17.10 \\
\hline Proposed method 2 (FOM) & 95.5 & 16.95 \\
\hline
\end{tabular}




\section{Conclusion and future work}

In this paper, in depth analysis of machine learning of Haar features with the respect to its various parameters has been performed. The proposed work for Haarfeatures training has been done on the basis of modified Viola-Jones training and detection framework. A number of modifications have been exercised in the original framework to find the scope of improvement in the overall detection framework. The basic algorithm of AdaBoost was implemented to generate the weak classifiers. The variations of training parameters have been studied for different training image's size. The detailed analysis of Haar features training by using example images of the size ranging from $14 \times 14$ to $30 \times 30$ pixels. Proposed system has been successfully tested on the benchmark datasets WIDER FACE, AFW and FDDB. The proposed work is found to be effective from the results computed on benchmark datasets in terms of training time and detection time.

The proposed system can further be improved by efficient Haar-features selection using statistical features analysis of other training parameters in prescreening stage. Based on this intuitive analysis more improvements can be exercised in the Haar features training process such as achieving features set reduction and improvement in time efficiency of the overall system. Further rotated Haar features can be integrated in the system for the detection of faces at various angular positions.

\section{Acknowledgment}

None.

\section{Conflicts of interest}

The authors have no conflicts of interest to declare.

\section{References}

[1] Viola P, Jones M. Rapid object detection using a boosted cascade of simple features. In proceedings of the 2001 computer society conference on computer vision and pattern recognition 2001. IEEE.

[2] Viola P, Jones MJ. Robust real-time face detection. International Journal of Computer Vision. 2004; $57: 137-54$.

[3] Egorov AD, Shtanko AN, Minin PE. Selection of viola-jones algorithm parameters for specific conditions. Bulletin of the Lebedev Physics Institute. 2015; 42:244-8.

[4] Mena AP, Mayoral MB, Diaz-lope E. Comparative study of the features used by algorithms based on viola and jones face detection algorithm. In international work-conference on the interplay between natural and artificial computation 2015 (pp. 175-83). Springer, Cham.
[5] Pandey S, Sharma S. An optimistic approach for implementing viola jones face detection algorithm in database system and in real time. International Journal of Engineering Research and Technology. 2015; 4(7):1118-22.

[6] Mutneja V, Singh S. Modified Viola-Jones algorithm with GPU accelerated training and parallelized skin color filtering-based face detection. Journal of RealTime Image Processing. 2019; 16:1573-93.

[7] Rusia MK, Singh DK, Ansari MA. Human face identification using lbp and haar-like features for real time attendance monitoring. In international conference on image information processing 2019 (pp. 612-6). IEEE.

[8] Papageorgiou CP, Oren M, Poggio T. A general framework for object detection. In sixth international conference on computer vision 1998 (pp. 555-62). IEEE.

[9] Lienhart R, Maydt J. An extended set of haar-like features for rapid object detection. In proceedings of the international conference on image processing 2002. IEEE.

[10] Saha S, Démoulin V. ALOHA: an efficient binary descriptor based on haar features. In international conference on image processing 2012 (pp. 2345-8). IEEE.

[11] Nirmalkar S, Janghel R. Haar features selection using statistics in boosting based object detection. 2012.

[12] Zhang S, Bauckhage $\mathrm{C}$, Cremers $\mathrm{AB}$. Informed haarlike features improve pedestrian detection. In proceedings of the IEEE conference on computer vision and pattern recognition 2014 (pp. 947-54).

[13] Wang YQ. An analysis of the Viola-Jones face detection algorithm. Image Processing On Line. 2014; 4:128-48.

[14] Murphy TM, Broussard R, Schultz R, Rakvic R, Ngo H. Face detection with a Viola-Jones based hybrid network. IET Biometrics. 2017; 6(3):200-10.

[15] Qezavati H, Majidi B, Manzuri MT. Partially covered face detection in presence of headscarf for surveillance applications. In international conference on pattern recognition and image analysis 2019 (pp. 195-9). IEEE.

[16] Chung BW, Park KY, Hwang SY. A fast and efficient Haar-like feature selection algorithm for object detection. The Journal of Korean Institute of Communications and Information Sciences. 2013; 38(6):486-91.

[17] Mutneja V, Singh S. Size-based performance analysis of haar-features for detection of facial images from low resolution surveillance videos. In international conference on intelligent computing, instrumentation and control technologies 2017 (pp. 1191-5). IEEE.

[18] Jin J, Zhu A, Wang Y, Wright J. A feature binding model in computer vision for object detection. Multimedia Tools and Applications. 2021; 80:1937797.

[19] Lin YN, Hsieh TY, Huang JJ, Yang CY, Shen VR, Bui HH. Fast Iris localization using haar-like features and AdaBoost algorithm. Multimedia Tools and Applications. 2020; 79:34339-62. 
[20] Adeshina SO, Ibrahim H, Teoh SS, Hoo SC. Custom face classification model for classroom using haar-like and LBP features with their performance comparisons. Electronics. 2021; 10(2):1-15.

[21] Pant DR, Sthapit R. Analysis of micro facial expression by machine and deep learning methods: haar, $\mathrm{CNN}$, and RNN. Journal of the Institute of Engineering. 2021; 16(1):95-101.

[22] Mordvintseva YA, Belitsky AA. Training the haar cascade classifier in sorting radio-electronic components. automating the collection of images for teaching. In conference of Russian young researchers in electrical and electronic engineering 2021 (pp. 101922). IEEE.

[23] Odaudu SN, Adedokun EA, Salaudeen AT, Marshall FF, Ibrahim Y, Ikpe DE. Sequential feature selection using hybridized differential evolution algorithm and haar cascade for object detection framework. Covenant Journal of Informatics and Communication Technology. 2020; 8(1):67-82.

[24] Wang YG, Li M, Ma Z, Montufar G, Zhuang X, Fan Y. Haar graph pooling. In international conference on machine learning 2020 (pp. 9952-62). PMLR.

[25] Wu H, Cao Y, Wei H, Tian Z. Face recognition based on haar like and euclidean distance. In journal of physics: conference series 2021 (pp. 1-7). IOP Publishing.

[26] Yang S, Luo P, Loy CC, Tang X. Wider face: a face detection benchmark. In proceedings of the conference on computer vision and pattern recognition 2016 (pp. 5525-33).IEEE.

[27] Zhu X, Ramanan D. Face detection, pose estimation, and landmark localization in the wild. In conference on computer vision and pattern recognition 2012 (pp. 2879-86). IEEE.

[28] Jain V, Learned-miller E. Fddb: a benchmark for face detection in unconstrained settings. UMass Amherst Technical Report; 2010:1-11.

[29] http://cbcl.mit.edu/software-datasets/FaceData2.html. Accessed 26 March 2015.

[30] Fuhl W, Eivazi S, Hosp B, Eivazi A, Rosenstiel W, Kasneci E. BORE: boosted-oriented edge optimization for robust, real time remote pupil center detection. In proceedings of the ACM symposium on eye tracking research \& applications 2018 (pp. 1-5).

[31] Fuhl W, Gao H, Kasneci E. Tiny convolution, decision tree, and binary neuronal networks for robust and real time pupil outline estimation. In ACM symposium on eye tracking research and applications 2020 (pp. 1-5).

[32] Fuhl W. 1000 pupil segmentations in a second using haar like features and statistical learning. arXiv preprint arXiv:2102.01921. 2021.

[33] Naido S, Porle RR. Face detection using colour and haar features for indoor surveillance. In 2nd international conference on artificial intelligence in engineering and technology 2020 (pp. 1-5). IEEE.

[34] Hou Y, Wang M. Face detection method based on improved convolutional neural network. In international workshop on electronic communication and artificial intelligence 2020 (pp. 102-5). IEEE.
[35] Naveen P, Sivakumar P. Pose and head orientation invariant face detection based on optimised aggregate channel feature. Annals of the Romanian Society for Cell Biology. 2021; 25(5):4368-90.

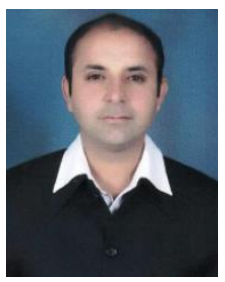

Dr. Vikram Mutneja is Associate Professor in the department of Electronics \& Communication Engineering, Shaheed Bhagat Singh, State University, Ferozepur (Punajb, India). He received his Bachelor's degree (B.Tech.) from Guru Nanak Dev University, Amritsar, Punjab (India) with specialization in Electronics \& Communication Engineering in year 1998, Master's degree (M.Tech.) from Giani Zail Singh College of Engineering \& Technology, Bathinda (Punjab) (India) under Punjab Technical University (PTU, Jalandhar) with first division in Electronics \& Communication Engineering in year 2008 and Doctoral degree (Ph.D.) from I.K. Gujral Punjab Technical University, Kapurthala (Punajb, India) in year 2018. He has industrial experience of around 5 years (1998-2003) in which he worked in diverse fields such as Hardware and Networking Technical Support, Software Development for Web and Embedded Systems. During his teaching experience of around 17 years (2003-2020) he worked mainly in the areas of image processing, artificial intelligence, embedded systems, VLSI and digital signal processing.

Email: vikram.mutneja@gmail.com

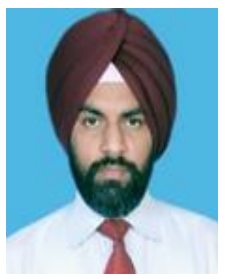

Dr. Satvir Singh received his Bachelor's degree (B.Tech.) from Dr. B. R. Ambedkar National Institute of Technology, Jalandhar, Punjab (India) with specialization in Electronics \& Communication Engineering in year 1998, Master's degree (M.E.) from Delhi Technological University (Formerly, Delhi College of Engineering), Delhi (India) with distinction in Electronics \& Communication Engineering in year 2000 and Doctoral degree (Ph.D.) from Maharshi Dayanand University, Rohtak, Haryana (India) in year 2011. He is a Fellow member of IE, IETE and Life Member of ISTE and IAENG. During his 17 years of teaching experience, he served as Assistant Professor and Head, Department of Electronics \& Communication Engineering at BRCM College of Engineering \& Technology, Bahal, (Bhiwani) Haryana, India and as Associate Professor \& Professor, Department of Electronics \& Communication Engineering at Shaheed Bhagat Singh State Technical Campus (Formerly, SBS College of Engineering \& Technology), Ferozepur Punjab, India. Presently, he is serving as Associate Professor at IK Gujral Punjab Technical University, Kapurthala in the Department of Electronics \& Communication Engineering. He has also handled the responsibilities of Estate Officer, Store \& Purchase Officer and TEQIP-II Procurement Coordinator. His fields of special interest include Evolutionary Algorithms, High Performance Computing, Type-1 \& Type-2 Fuzzy Logic 
Systems, Wireless Sensor Networks and Artificial Neural Networks for solving engineering problems. He has published nearly 75 research papers in International Journals and Conferences. He has delivered more than 60 Invited Talks during National and International Conferences, Seminar, Short Term Courses and Workshops. He completed two AICTE funded projects under MODROB Scheme worth 15 Lacs. He has also conducted four Faculty Development Programmes, of total duration of six weeks, around Soft Computing techniques under various schemes of AICTE and TEQIP. He has guided 05 Ph.D. and 15 M.Tech. research scholars and, at present, $05 \mathrm{Ph} . \mathrm{D}$. research scholars are conducting research under his guidance.

Email: drsatvir.in@gmail.com

\begin{tabular}{|c|c|c|}
\hline \multicolumn{3}{|c|}{ Appendix I } \\
\hline S.No. & Abbreviations & Description \\
\hline 1 & AFW & Wider Face \\
\hline 2 & ALOHA & Aggregated LOcal Haar \\
\hline 3 & BW & Bandwidth \\
\hline 4 & CBCL & $\begin{array}{l}\text { Center for Biological \& } \\
\text { Computational Learning }\end{array}$ \\
\hline 5 & $\mathrm{CNN}$ & Convolution Neural Network \\
\hline 6 & CUDA & $\begin{array}{l}\text { Compute Unified Device } \\
\text { Architecture }\end{array}$ \\
\hline 7 & cType & Classifier type \\
\hline 8 & $\mathrm{DE}$ & Differential Evolution \\
\hline 9 & $E_{p}$ & Positive Error \\
\hline 10 & $E_{t}$ & Total Error \\
\hline 11 & FAR & False Alarm Rate \\
\hline 12 & FDDB & $\begin{array}{l}\text { Face Detection Dataset and } \\
\text { Benchmark }\end{array}$ \\
\hline 13 & FDR & False Detection Rate \\
\hline 14 & FOM & Figure of Merit \\
\hline 15 & $\mathrm{~F}_{\mathrm{P}}$ & False face detections \\
\hline 17 & $\mathrm{~F}_{\mathrm{N}}$ & False non-face detections \\
\hline 18 & GPGPU & $\begin{array}{l}\text { General-Purpose } \\
\text { Processing Unit }\end{array}$ \\
\hline 19 & GPU & Graphical Processor Unit \\
\hline 20 & LBP & Local Binary Patterns \\
\hline 21 & LBPH & $\begin{array}{ll}\text { Local Binary Patterns } \\
\text { Histogram }\end{array}$ \\
\hline 22 & $\mathrm{~L}_{\mathrm{L}}$ & $\begin{array}{l}\text { Lower limit of threshold of } \\
\text { active window of a selected } \\
\text { Haar classifier }\end{array}$ \\
\hline 23 & RNN & Recurrent Neural Network \\
\hline 24 & SVM & Support Vector Machine \\
\hline 25 & TFD & Total false detections \\
\hline 26 & TDR & True Detection Rate \\
\hline 27 & TPR & True Positive Rate \\
\hline 28 & tpMax & $\begin{array}{l}\text { Maximum value of threshold } \\
\text { of positive training images }\end{array}$ \\
\hline 29 & ThRange & $\begin{array}{l}\text { Threshold range of active } \\
\text { window of a selected Haar } \\
\text { classifier }\end{array}$ \\
\hline 30 & tpMin & $\begin{array}{l}\text { Minimum value of threshold } \\
\text { of positive training images }\end{array}$ \\
\hline 31 & tpMean & $\begin{array}{l}\text { Mean value of threshold of } \\
\text { positive training images }\end{array}$ \\
\hline 32 & $\mathrm{~T}_{\mathrm{P}}$ & True face detection \\
\hline 33 & $\mathrm{~T}_{\mathrm{N}}$ & True non-face detections \\
\hline 34 & TTD & Total true detections \\
\hline 35 & $\mathrm{U}_{\mathrm{L}}$ & $\begin{array}{l}\text { Upper limit of threshold of } \\
\text { active window of a selected } \\
\text { Haar classifier }\end{array}$ \\
\hline 36 & $\mathrm{w}, 1$ & $\begin{array}{l}\text { Width and length of Haar } \\
\text { feature }\end{array}$ \\
\hline 37 & $\mathrm{x}, \mathrm{y}$ & $\begin{array}{l}x \& y \text { co-ordinates of Haar } \\
\text { feature }\end{array}$ \\
\hline
\end{tabular}

